\title{
ZONEAMENTO AMBIENTAL APLICADO À CONSERVAÇÃO DO SOLO E DA ÁGUA ${ }^{(1)}$
}

\author{
Paulo Tarso Sanches de Oliveira ${ }^{(2)}$, Teodorico Alves Sobrinho ${ }^{(3)}$, \\ Dulce Buchala Bicca Rodrigues ${ }^{(4)}$ \& Elói Panachuki ${ }^{(5)}$
}

\begin{abstract}
RESUMO
O zoneamento ambiental auxilia na gestão do solo e da água, pois fornece informações de uso e manejo do solo de forma adequada. Assim, propõe-se neste estudo: (i) a aplicação do zoneamento ambiental, baseado em valores de perda de solo, tolerância à perda de solo e à legislação ambiental; e (ii) etapa de monitoramento das características quali-quantitativas dos cursos d'água a partir do uso de curvas Total Maximum Daily Loads (TMDL). O estudo foi realizado na bacia hidrográfica do ribeirão Salobra, localizada na transição entre os biomas Cerrado e Pantanal. A partir do cruzamento dos planos de informação, obteve-se a divisão da área em cinco zonas de uso do solo, determinadas de acordo com a conservação e preservação da vegetação nativa, ocorrência de áreas úmidas e nascentes, uso adequado do solo e recuperação de áreas erodidas. Paralelamente, as curvas TMDL foram obtidas a partir da curva de permanência e de padrões de qualidade da água. Verificou-se que cerca de $6 \%$ da área em estudo apresentou perda de solo superior ao limite tolerável e foi definida como área prioritária de recuperação no contexto do planejamento ambiental. $O$ método aplicado permitiu a determinação de zonas específicas de uso do solo, identificação de irregularidades e de prioridades de conservação do solo. Além disso, as curvas TMDL constituem boa alternativa de monitoramento da água, pois produzem informações em condições críticas do corpo d'água.
\end{abstract}

Termos de indexação: erosão hídrica, TMDLs, regionalização de vazão.

\footnotetext{
(1) Parte da Dissertação de Mestrado do primeiro autor. Recebido para publicação em 7 de fevereiro de 2011 e aprovado em 6 de junho de 2011.

(2) Doutorando em Hidráulica e Saneamento na Universidade de São Paulo - USP. Caixa Postal 359, CEP 13560-970 São Carlos (SP). E-mail: paulot@sc.usp.br

(3) Professor Associado III da Universidade Federal de Mato Grosso do Sul, CCET/UFMS. E-mail: talves@ufms.br

${ }^{(4)}$ Doutoranda em Hidráulica e Saneamento na Universidade de São Paulo - USP. Caixa Postal 359, CEP 13560-970 São Carlos (SP). E-mail: dulce@sc.usp.br

(5) Professor da Universidade Estadual de Mato Grosso do Sul - UEMS. Caixa Postal 25, CEP 79.200-000 Aquidauana (MS). Email: eloip@uems.br
} 


\title{
SUMMARY: ENVIRONMENTAL ZONING APPLIED TO SOIL AND WATER CONSERVATION
}

\begin{abstract}
The environmental zoning for soil and water conservation provides useful information about land use and management. Thus, this study proposes (i) an environmental zoning, based on the combination of Universal Soil Loss Equation (USLE), soil loss tolerance (T) estimates adapted to Brazilian soils and environmental legislation; and (ii) qualitativequantitative monitoring of water resources using Total Maximum Daily Load curves (TMDLs). The study area was the Ribeirão Salobra Basin (Terenos, MS, Brazil), located in the transition between the biomes Cerrado and Pantanal. Several information plans were crossed resulting in the division of the total area into five zones with different land use profiles, determined according to the conservation and preservation of native vegetation, occurrence of wet areas and springs, adequate land use and eroded area recovery. In a next step, the TMDL curves were obtained from duration curves and water quality standards. In about $6 \%$ of the study area, soil losses exceeded the threshold, defining areas of priority to be recovered in the focus of environmental planning. The proposed methodology was suited for the determination of specific land use zones, as well as the identification of conservation priorities and irregularities. Furthermore, the TMDL curves indicate critical conditions of water bodies and represent an alternative tool for water monitoring.
\end{abstract}

Index terms: water erosion, TMDLs, regionalization of water flow.

\section{INTRODUÇÃO}

A erosão hídrica ocasiona diversos efeitos prejudiciais ao solo e à água que envolvem desde aspectos de perda de produtividade agrícola até impactos sobre os recursos hídricos. Assim, a predição de perda de solo é importante para avaliar os riscos de erosão do solo e determinar seus adequados usos e manejos (Oliveira et al., 2011). Nesse sentido, muitos modelos matemáticos (empírico, conceitual e baseados em processo) têm sido desenvolvidos para estimativa da erosão do solo em diferentes escalas espaciais e temporais (Moehansyah et al., 2004; Ferro, 2010). Os modelos variam desde procedimentos complexos, que requerem uma série de parâmetros de entrada, como Water Erosion Prediction Project (WEPP) (Nearing et al., 1989), Kinematic Runoff and Erosion (KINEROS) (Woolhiser et al., 1990) e European Soil Erosion Model (EUROSEM) (Morgan et al., 1998), até métodos mais simplificados, como Universal Soil Loss Equation (USLE) (Wischmeier \& Smith, 1978), Revised Universal Soil Loss Equation (Renard et al., 1997) e Morgan-Morgan and Finney (MMF) (Morgan, 2001).

Modelos que requerem vários parâmetros de entrada podem ser inviáveis para aplicação em locais com ausência de dados ou de dificil acesso, como em várias regiões do Brasil. Nesse sentido, diversos autores consideram que a USLE representa excelente modelo de predição da perda de solo, em virtude da sua aplicabilidade e da confiabilidade dos valores de perda de solo estimados (Risse et al., 1993; Ferro, 2010). A aplicação da USLE em escala de bacias hidrográficas tem sido facilitada pelo uso de Sistema de Informações Geográficas (SIG), aprsentando-se, assim, como boa ferramenta para o planejamento conservacionista do solo e da água (Weill \& Sparovek, 2008; Beskow et al., 2009; Oliveira et al., 2011).

Os valores de perda de solo, para a média anual, estimados pela USLE, podem ser comparados com os de tolerância à perda de solo (T), com o intuito de verificar áreas de risco de erosão e subsidiar o adequado uso e manejo do solo. Desse modo, diversos autores utilizaram valores do risco de erosão no planejamento agrícola e ambiental (Zhang et al., 2004; Basic et al., 2004; Bhattacharyya et al., 2008; Oliveira et al., 2011). Oliveira et al. (2011) desenvolveram um método de zoneamento ambiental aplicado em escala de bacias hidrográficas, a partir de valores de risco de erosão e de uso do solo permissível, e adequado à legislação ambiental, concluindo que o método pode ser empregado, de forma satisfatória, em outras bacias hidrográficas.

A partir da implementação do zoneamento ambiental, torna-se fundamental o conhecimento de ferramentas que auxiliem no monitoramento do uso e manejo do solo e das características qualiquantitativas dos cursos d'água. No monitoramento das mudanças de uso do solo, podem-se utilizar informações de sensoriamento remoto processadas em Sistemas de Informações Geográficas (SIG). Com relação às características quali-quantitativas dos cursos d'água, neste trabalho sugeriu-se o uso de curvas Total Maximum Daily Loads (TMDLs).

O conhecimento da carga máxima de poluentes permitida para determinado curso d'água em distintos períodos constitui a maneira eficaz de verificar o atendimento à legislação, a qual é aplicada em alguns países a partir de curvas TMDL (Havens \& Schelske, 
2001; Borsuk et al., 2002; Kang et al., 2006; Stringfellow et al., 2009). Conforme Zhang \& Yu (2008), a utilização de curvas de TMDL é estratégia importante para atingir as metas de qualidade da água em gestão de bacias hidrográficas. Segundo Ogg \& Keith (2002), as curvas TMDL ainda auxiliam na análise das fontes de poluição (pontual, difusa, natural ou antrópica), capacidade de assimilação do poluente e implementação de medidas de controle. Apesar da aceitação e aplicação de curvas TMDL em vários países, no Brasil essa abordagem ainda é pouco desenvolvida no âmbito da gestão das águas.

Assim, propuseram-se neste trabalho: (i) a aplicação do zoneamento ambiental, baseado em valores de perda de solo, tolerância à perda de solo e à legislação ambiental; e (ii) etapa de monitoramento das características quali-quantitativas dos cursos d'água a partir do uso de curvas TMDL.

\section{MATERIAL E MÉTODOS}

\section{Área de estudo}

O estudo foi realizado tomando-se por base a bacia hidrográfica do ribeirão Salobra, com área aproximada de $540 \mathrm{~km}^{2}$, localizada entre as coordenadas de latitudes $20^{\circ} 12^{\prime} \mathrm{S}$ a $20^{\circ} 28^{\prime} \mathrm{S}$ e longitudes $54^{\circ} 55^{\prime} \mathrm{W}$ a $55^{\circ} 16^{\prime} \mathrm{W}$, em uma área de transição entre os biomas Cerrado e Pantanal (Figura 1). O ribeirão Salobra é tributário do rio Aquidauana, importante curso d'água para suprimento das atividades pesqueiras e agrícolas na região.

De acordo com a classificação de Köppen, o clima na região situa-se na fase de transição entre Cfa, mesotérmico úmido, sem estiagem e AW tropical úmido, com uma estação chuvosa no verão e uma de seca no inverno. A precipitação e a temperatura média anual correspondem a $1.500 \mathrm{~mm}$ e $23^{\circ} \mathrm{C}$, respectivamente. A altitude na área varia de 200 a $400 \mathrm{~m}$, e o relevo é predominantemente plano, com declividade média de $2 \%$.

\section{Universal Soil Loss Equation (USLE)}

A USLE foi implementada no software SIG ArcGis (ESRI, 2006) para estimativa da perda de solo por erosão laminar e em sulcos, conforme equação 1 (Wischmeier \& Smith, 1978).

$$
\mathrm{A}=\mathrm{R} \text { K L S C P }
$$

em que $\mathrm{A}=$ perda média anual de solo por unidade de área $\left(\mathrm{t} \mathrm{ha}^{-1} \mathrm{ano}^{-1}\right), \mathrm{R}=$ erosividade da chuva (MJ mm ha-1 $\mathrm{h}^{-1}$ ano-1 $^{-1}$, $\mathrm{K}=$ erodibilidade do solo (t h MJ-1 $\mathrm{mm}^{-1}$ ), LS = fator topográfico (adimensional), $\mathrm{C}=$ uso e manejo do solo (adimensional) e $\mathrm{P}=$ práticas conservacionistas (adimensional).

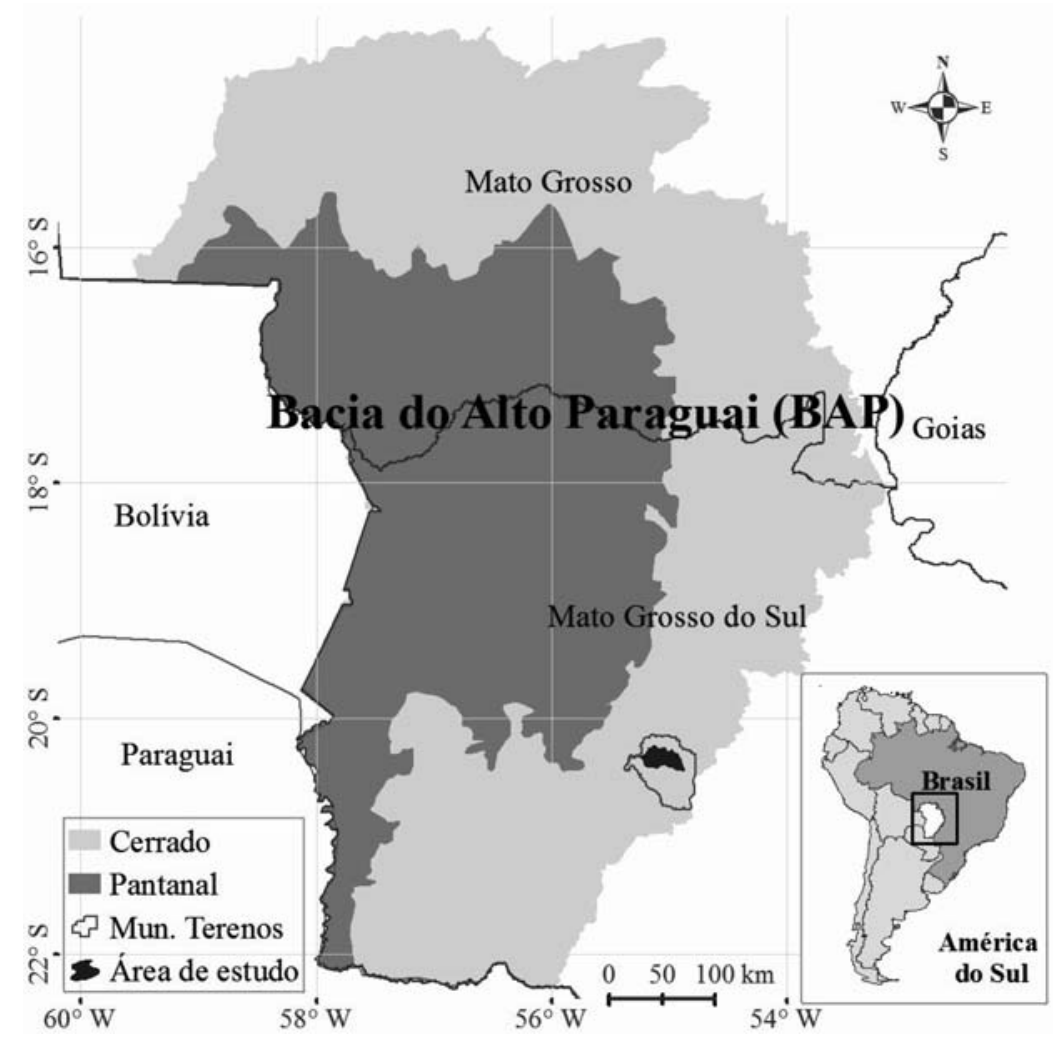

Figura 1. Área de estudo. 


\section{Erosividade média anual}

Os resultados de erosividade média anual foram obtidos de Oliveira (2011). Esse autor utilizou dados provenientes de três estações pluviográficas, localizadas nos municípios de Campo Grande, Dourados e Coxim. Além disso, coletou dados de 109 estações pluviométricas localizadas no Estado de Mato Grosso do Sul, a partir do Sistema de Informações Hidrológicas da Agência Nacional de Águas (HidroWeb). O mapa do índice de erosividade foi gerado pelo citado autor a partir do ajuste do semivariograma, do processo de validação cruzada e da aplicação da krigagem ordinária.

Assim, neste trabalho realizou-se o recorte do mapa de erosividade do Estado de Mato Grosso do Sul, conforme o limite da área em estudo.

\section{Erodibilidade e tolerância à perda de solo}

$\mathrm{O}$ fator de erodibilidade do solo (fator $\mathrm{K}$ ) e a tolerância à perda de solo $(\mathrm{T})$ foram obtidos a partir das características descritas nos mapas de solo, escalas 1:1.000.000 (Brasil, 1982) e 1:250.000 (PCBAP, 1997). A cada classe de solo verificada na área em estudo foram atribuídos valores de $\mathrm{K}$ e $\mathrm{T}$, conforme os resultados de Oliveira et al. (2011) para solos da região e com as características semelhantes às da área estudada.

\section{Fator topográfico}

Para obtenção do fator topográfico foi gerado um Modelo Digital de Elevação (MDE) a partir da base topográfica, referente às folhas Campo Grande SF. 21-X-B-II (DSG, 1979) e Palmeiras SF. 21-X-B-I (DSG, 1988), ambas na escala 1:100.000. O MDE foi desenvolvido no software SPRING 4.3.3 (Câmara, 1996), com resolução de 30 x 30 m.

O MDE gerado foi processado no software USLE2D para o cálculo do fator topográfico. O fator L é calculado por meio da divisão da área de contribuição pela largura ao longo do fluxo, que pode passar dentro de uma célula. Essa largura depende da direção do fluxo e é calculada utilizando o aspecto de direção (Desmet \& Govers, 1996; Oliveira et al., 2010) (Equação 2).

$$
\mathrm{L}_{\mathrm{i}, \mathrm{j}}=\left[\left(\mathrm{A}_{\mathrm{i}, \mathrm{j}}+\mathrm{D}^{2}\right)^{\mathrm{m}+1}-\left(\mathrm{A}_{\mathrm{i}, \mathrm{j}}\right)^{\mathrm{m}+1}\right] /\left[\mathrm{x}_{\mathrm{i}, \mathrm{j}}^{\mathrm{m}} \mathrm{D}^{\mathrm{m}+2}(22,13)^{\mathrm{m}}\right]
$$

em que $\mathrm{L}_{\mathrm{i}, \mathrm{j}}=$ fator de comprimento de vertente de uma célula com coordenadas ( $i, j$ ), $A_{i, j}=$ área de contribuição de uma célula com coordenadas $(i, j)\left(m^{2}\right)$, $\mathrm{D}=$ tamanho da grade de células $(\mathrm{m}), \mathrm{x}_{\mathrm{i}, \mathrm{j}}=$ valor da direção do fluxo e $\mathrm{m}=$ coeficiente dependente $\mathrm{da}$ declividade.

O expoente $(\mathrm{m})$ da equação 1 foi calculado de acordo com Wischmeier \& Smith (1978), sendo $\mathrm{S}<1 \%, \mathrm{~m}=$ 0,$2 ; 1 \% \leq \mathrm{S} \leq 3 \%, \mathrm{~m}=0,3 ; 3 \%<\mathrm{S} \leq 5 \%, \mathrm{~m}=0,4 ; \mathrm{e}$ $\mathrm{S}>5 \%, \mathrm{~m}=0,5$. Utilizou-se a equação 3 proposta por Wischmeier \& Smith (1978) para o cálculo do fator S.

$$
\mathrm{S}_{\mathrm{ws}}=65,41 \operatorname{sen}^{2} \theta+4,56 \operatorname{sen} \theta+0,065
$$

sendo $\theta=$ ângulo (em graus) da encosta.

\section{Fatores uso e manejo do solo e práticas conser- vacionistas}

O mapa de uso do solo foi obtido a partir da classificação da imagem digital do sensor Thematic Mapper (TM) do satélite LANDSAT 5, com data de passagem de 2008, referente à órbita/ponto 225/74 (INPE, 2008). No processo de classificação da imagem, realizou-se o procedimento de segmentação e classificação supervisionada por regiões, disponível no SPRING 4.3.3. Desse modo, foram divididas em sete classes de uso e ocupação do solo, sendo-lhes atribuídos valores do fator C, obtidos de Oliveira et al. (2011). Ao fator $\mathrm{P}$ atribui-se o valor 1, considerando a não adoção de práticas conservacionistas em toda a área em estudo.

\section{Zoneamento ambiental}

O critério utilizado para a realização do zoneamento ambiental foi o de conservação do solo e da água. Assim, realizou-se a integração dos planos de informação: uso permissível do solo $\left(\mathrm{CP}_{\mathrm{p}}\right)$; risco de erosão (ER); uso atual do solo; e legislação ambiental referente à Área de Preservação Permanente (APP), conforme proposto por Oliveira et al. (2011).

A ocupação atual do solo foi utilizada visando à identificação e proposição de conservação e à preservação das áreas de vegetação nativa e áreas úmidas, denominadas Zona 1 . A Zona 2 foi definida como áreas de recomposição de APPs, ou seja, áreas que deveriam ser APP, mas atualmente são ocupadas de forma irregular. Para tanto, as APPs foram definidas de acordo com a legislação vigente - Lei $n$. 4771/1965 (Brasil, 1965) e Resoluções CONAMA n ${ }^{\circ}$ s 302/2002 (Brasil, 2002a) e 303/2002 (Brasil, 2002b).

A razão entre a perda de solo e os valores de tolerância de perda de solo foi realizada com o intuito de verificar as áreas onde essas perdas ocorriam acima do valor tolerável para cada tipo de solo. Essa razão é denominada risco de erosão (ER) (Basic et al., 2004; Oliveira et al., 2011) (Equação 4).

$$
\mathrm{ER}=\mathrm{A} / \mathrm{T}
$$

O ER foi utilizado com o intuito de identificar as áreas que se encontravam degradadas pela erosão e necessitavam de recuperação imediata. Assim, essas áreas foram definidas como Zona 3. Para compor essa classe, utilizaram-se os valores de ER $>1$, ou seja, aqueles cuja perda de solo estimada é superior à perda de solo tolerável (Oliveira et al., 2011).

De acordo com Wischmeier \& Smith (1978), o valor de perda de solo (A) da USLE pode ser substituído pela perda de solo tolerável (T), dado em t ha-1 ano-1. Assim, considerando o valor de $\mathrm{P}=1$, pode-se 
determinar a capacidade de uso e manejo permissível do solo (Equação 5).

$$
\mathrm{CP}_{\mathrm{p}}=\mathrm{T} / \mathrm{R} \mathrm{KLS}
$$

A partir dessa equação, é possível estabelecer e analisar áreas de ocupação do solo, considerando-se esse índice como indicador do provável ajuste da ocupação do solo de acordo com as características ambientais que influenciam nos processos erosivos. Assim, os valores de $\mathrm{CP}_{\mathrm{p}}$ foram divididos em duas classes de uso agrícola, correspondendo às Zonas $4 \mathrm{e}$ 5. Na Zona $4, \mathrm{o} \mathrm{CP}_{\mathrm{p}}$ foi agrupado em valores variando de 0,1 a 1,0. Nessa zona é permitido o uso de culturas perenes e anuais com manejo e de áreas de pastagem. $\mathrm{Na}$ Zona 5 , os valores de $\mathrm{CP}_{\mathrm{p}}$ variam de 0 a 0,1 . Essas áreas devem ser ocupadas por boa cobertura do solo e manejo, pois apresentam maior risco natural de erosão. Assim, é possível a ocupação do solo por culturas perenes e por pastagens, com manejo regular (Oliveira et al., 2011).

Após a obtenção de cada zona, realizaram-se o agrupamento e a quantificação das zonas, formando o mapa de zoneamento ambiental proposto por Oliveira et al. (2011). No quadro 1, encontra-se o resumo das zonas definidas para uso do solo.

\section{Quadro 1. Zoneamento ambiental}

\begin{tabular}{cl}
\hline Zona & \multicolumn{1}{c}{ Denominação } \\
\hline 1 & Manutenção e preservação da vegetação e áreas úmidas \\
2 & Recuperação de APP (definida conforme a legislação vigente) \\
3 & Recuperação de áreas degradadas por erosão do solo (ER $>1$ ) \\
4 & Uso agrícola destinado a culturas anuais (áreas resistentes) \\
5 & Uso agrícola para pastagens com manejo regular (áreas frágeis)
\end{tabular}

Fonte: Adaptado de Oliveira et al. (2011).

\section{Proposta de monitoramento das caracterís- ticas quali-quantitativas da água}

Neste estudo foi utilizado o método de regionalização de vazão, pois não se dispunha de dados de séries históricas de vazão. Para tanto, foram utilizadas quatro estações fluviométricas que envolviam a área em estudo, com período de dados superior a dez anos, localizadas nos rios Aquidauana e Paraguai. Os dados dessas estações são provenientes do Sistema de Informações Hidrológicas HidroWeb (ANA, 2010), cujas principais características estão apresentadas no quadro 2.

As curvas de permanência foram desenvolvidas conforme método descrito em Tucci $(2002,2003)$ e dividida da seguinte forma: seleção da série de dados de vazões diárias a ser utilizada para a obtenção da curva de permanência; definição de 50 intervalos de classe das vazões diárias; subdivisão dos intervalos de classe com base na escala logarítmica devido à
Quadro 2. Principais características das estações fluviométricas

\begin{tabular}{lrlr}
\hline \multicolumn{1}{c}{ Nome } & Código-ANA & \multicolumn{1}{c}{ Rio } & Área \\
\hline & & & $\mathrm{km}^{2}$ \\
Ponte do Grego & 66926000 & Aquidauana & 4.890 \\
Palmeiras & 66941000 & Aquidauana & 11.200 \\
Aquidauana & 66945000 & Aquidauana & 15.200 \\
Barranco Branco & 67030000 & Paraguai & 453.000 \\
\hline
\end{tabular}

grande variação de magnitude das vazões envolvidas; e cálculo do intervalo de classe $(\Delta \mathrm{X})$ pela equação 6 .

$$
\Delta X=\frac{\left[\ln \left(Q_{\text {max }}\right)-\ln \left(Q_{\text {min }}\right)\right]}{50}
$$

em que $Q_{\text {máx }}=$ vazão máxima da série e $Q_{\text {mín }}=$ vazão mínima da série.

O cálculo dos limites dos intervalos, a partir de $\mathrm{Q}_{\text {mín }}$ e adicionando o intervalo calculado anteriormente, resultou na vazão do limite superior do intervalo $i$ (Equação 7).

$$
Q_{\mathrm{i}+1}=\operatorname{esp}\left[\ln \left(Q_{\mathrm{i}}\right)+\Delta \mathrm{X}\right]
$$

A determinação, com base nos dados de vazão da série histórica de cada estação fluviométrica, do número de vazões classificadas em cada intervalo e a obtenção da frequência (fi) associada ao limite inferior de cada intervalo ocorreram pela equação 8 .

$$
f_{i}=\frac{N_{q i}}{N T} \times 100
$$

em que $\mathrm{N}_{\mathrm{qi}}=$ número de vazões de cada intervalo e $\mathrm{NT}=$ número total de vazões.

A partir das curvas de permanência calculadas, realizou-se a regionalização das vazões $\mathrm{Q}_{50}, \mathrm{Q}_{90} \mathrm{e} \mathrm{Q}_{95}$. Assim, utilizou-se o método tradicional de regionalização que envolve a definição prévia das regiões hidrologicamente homogêneas e a obtenção das equações que permitem associar a vazão com variáveis físicas e climáticas das bacias hidrográficas (Tucci, 2002). No processo de regionalização de vazão, empregou-se o Sistema Computacional para a Regionalização de Vazões (SisCORV) (Sousa, 2009). Para a obtenção dos diversos modelos de equações de regionalização, foram realizados processos de regressão linear múltipla e a estimativa da variância do erro no software SisCORV.

Pela análise de regressão, verificou-se como uma ou mais variáveis se relacionam, ou seja, como as variáveis independentes influenciam as variáveis dependentes. A função matemática que relaciona as variáveis é obtida conforme a equação 9 .

$$
\mathrm{Q}=\mathrm{F}(\mathrm{X} 1, \mathrm{X} 2, \mathrm{X} 3, \mathrm{X} 4, \ldots \mathrm{Xn})
$$


em que $\mathrm{Q}$ = vazão estimada, $\mathrm{X}$ = variável independente e $\mathrm{n}=$ número de variáveis independentes.

Os melhores modelos resultantes da aplicação da regressão múltipla foram selecionados, observandose os maiores valores do coeficiente de determinação ajustado e resultados significativos pelo teste $\mathrm{F}$ a $95 \%$.

A regionalização da curva de permanência foi desenvolvida conforme o método descrito em Tucci (2002, 2003), para ser utilizada na etapa proposta de monitoramento das características quali-quantitativas da água na foz do ribeirão Salobra. Conforme Tucci (2002), o ajuste da curva de permanência a uma função exponencial geralmente apresenta resultados satisfatórios somente na faixa dos pontos utilizados para seu ajuste (Equação 10).

$$
\mathrm{Q}=\exp (\mathrm{aP}+\mathrm{b})
$$

em que $\mathrm{P}=$ permanência ( 0 a 1 ) e a e b são obtidos a partir dos valores característicos da $\mathrm{Q}_{50}$ e $\mathrm{Q}_{95}$ (equações 11 e 12).

$$
\begin{gathered}
a=-\frac{\ln \left(\frac{Q_{50}}{Q_{95}}\right)}{0,45} \\
b=\ln Q_{50}-0,50 a
\end{gathered}
$$

A curva de permanência para o ponto localizado na foz do ribeirão Salobra foi utilizada para a obtenção das curvas de Total Maximum Daily Loads (TMDLs) referente a fósforo total (ambiente lótico) e nitrato. Essas curvas foram obtidas pelo produto entre as vazões da curva de permanência e os padrões de qualidade da água de rios Classe 2, definidos na Resolução CONAMA n. 357 (Brasil, 2005). Assim, os valores da abscissa são as permanências das vazões e os valores da ordenada, os valores de carga medidos no ponto de amostragem. Então, a curva-limite representa a carga permitida, ou seja, a TMDL para cada medida de vazão. Desse modo, podem-se plotar valores de qualidade da água e de vazão obtidos em todo o período de monitoramento, verificando, assim, o atendimento aos padrões de qualidade da água.

\section{RESULTADOS E DISCUSSÃO}

O valor médio de erosividade $( \pm \mathrm{sd})$ calculado da área de estudo foi de $9.872 \pm 266 \mathrm{MJ} \mathrm{mm} \mathrm{ha}^{-1} \mathrm{~h}^{-1} \mathrm{ano}^{-1}$. Comparando esse valor com os resultados de Oliveira (2011) para o Estado de Mato Grosso do Sul (9.274 1.210 $\mathrm{MJ} \mathrm{mm} \mathrm{ha}^{-1} \mathrm{~h}^{-1} \mathrm{ano}^{-1)}$, pode-se considerar que a área de estudo apresentava erosividade média. No entanto, notou-se que os valores de erosividade aumentaram no sentido norte da bacia hidrográfica, justamente nos locais onde existem solos com maior erodibilidade e valores elevados do fator topográfico (Figura 2a-c). (a)

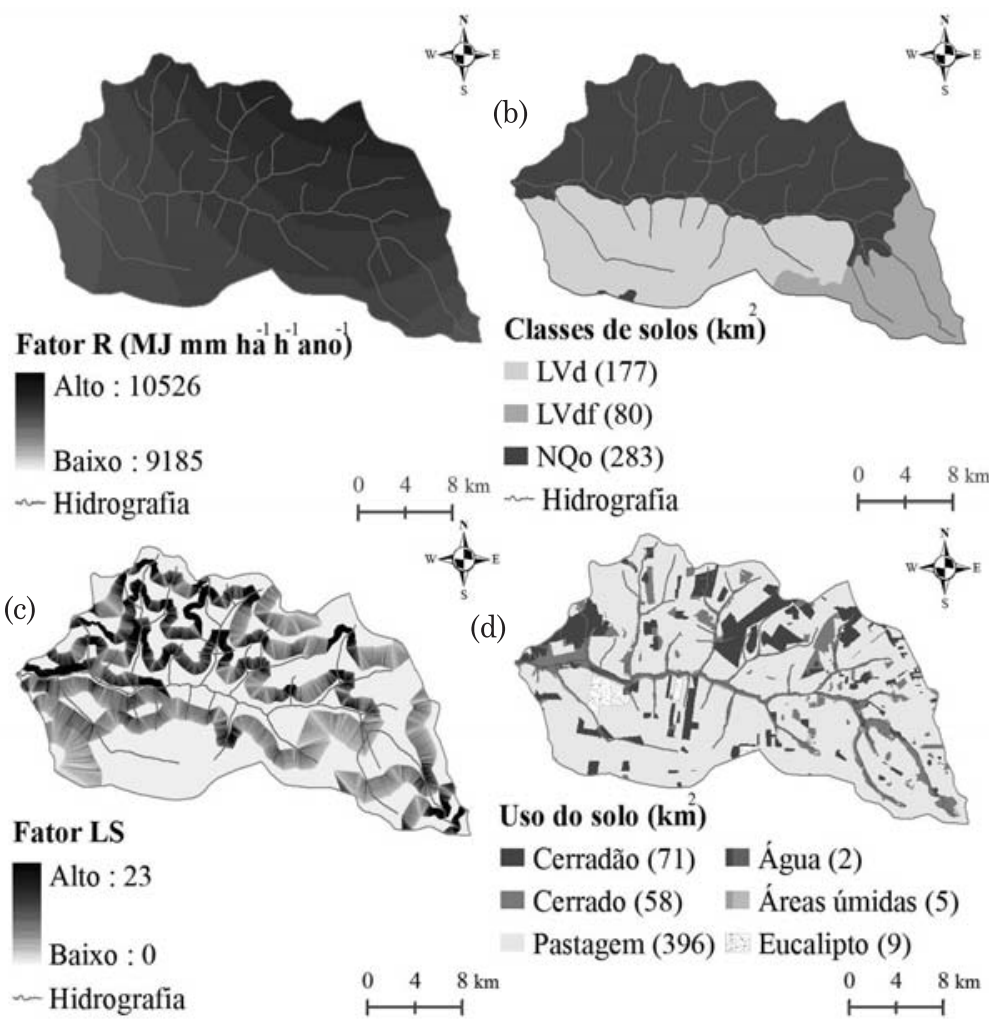

Figura 2. Dados obtidos para integração no modelo USLE: erosividade (fator R) (a); erodibilidade (fator K) (b); fator topográfico (LS) (c); e uso e manejo do solo (fator C) (d). 
A erodibilidade do solo na área em estudo pode ser classificada de forma decrescente, com os correspondentes percentuais de área, como: Neossolo Quartzarênico órtico (NQo) (52 \%), Latossolo Vermelho distrófico (LVd) (33\%) e Latossolo Vermelho distroférrio (LVdf) (15\%). Os solos com maior valor de tolerância de perda de solo (T) foram LVd, LVdf e NQo (Figura 2B e Quadro 3).

\section{Quadro 3. Classes de solo}

\begin{tabular}{lcc}
\hline \multicolumn{1}{c}{ Classes de solo } & Erodibilidade (K) & Tolerância (T) \\
\hline & $\mathrm{t} \mathrm{h} \mathrm{MJ}^{-1} \mathrm{~mm}^{-1}$ & $\mathrm{t} \mathrm{ha}^{-1} \mathrm{ano}^{-1}$ \\
$\begin{array}{l}\text { Neossolo Quatzarênico } \\
\text { órtico (NQo) }\end{array}$ & 0,0270 & 8,2 \\
$\begin{array}{l}\text { Latossolo Vermelho } \\
\text { distrófico (LVd) }\end{array}$ & 0,0130 & 15,0 \\
$\begin{array}{l}\text { Latossolo Vermelho } \\
\text { distroférrico (LVdf) }\end{array}$ & 0,0128 & 13,0 \\
\hline
\end{tabular}

Fonte: Adaptado de Oliveira et al. (2011).

O uso do solo na área em estudo era constituído, predominantemente, de áreas de pastagens $(73,2 \%)$, cerradão (13,1 \%), cerrado (10,7 \%), eucalipto (1,7 \%), área úmida $(0,9 \%)$ e água $(0,4 \%)$ (Figura $2 \mathrm{~d}$ ). Somando-se as classes de cerrado e cerradão, é possível verificar que $23,8 \%$ da área possuía cobertura de vegetação nativa. A pecuária de corte extensiva era a atividade agrícola predominante na área. No entanto, verificou-se a existência de pequenas propriedades rurais, nas quais ocorria a criação de gado leiteiro e de outros animais, como suínos, caprinos e ovinos. Outra forma de uso do solo na bacia hidrográfica era proveniente da atividade de piscicultura, sendo esta desenvolvida em grande parte pelo desvio ou represamento da água dos córregos para abastecimento dos tanques.

\section{Uso do solo permissível e risco de erosão}

O valor médio de perda de solo ( \pm sd) estimado na área de estudo foi de $2,1 \pm 4,7 \mathrm{t} \mathrm{ha}^{-1}$ ano $^{-1}$. Verificouse que $88,7 \%$ dos resultados correspondiam à perda de solo inferior a 5 t ha $^{-1}$ ano $^{-1}$. No entanto, os demais dados representam elevada perda de solo, sendo esta concentrada na região Norte da bacia hidrográfica (Figura 3a). É importante salientar que os valores estimados pela USLE apresentam algumas incertezas. Alguns autores relatam que o modelo pode superestimar pequenos valores de perda anual e subestimar elevados valores (Risse et al., 1993; Kinnell, 2010; Oliveira et al., 2011), embora isso também ocorra com modelos baseados em processos, como o Water Erosion Prediction Project (WEPP) (Tiwari et al., 2000; Amore et al., 2004). No entanto, a perda de solo estimada por longo período de tempo (e uma grande área) pode ser estimada corretamente com base no método estabelecido na USLE, pois superestimativas e subestimativas podem compensar- se mutuamente, resultando em boa avaliação geral da perda média anual de solo (Gabriels et al., 2003). Assim, os valores dessa perda obtidos neste estudo podem ser utilizados para aplicação do zoneamento ambiental de forma satisfatória.

Os resultados obtidos do uso permissível do solo (Figura $3 b$ ) indicam que para valores próximos a zero se devem priorizar a manutenção da cobertura vegetal e o manejo regular do solo. Já valores superiores a 0,5 evidenciam que a área é mais resistente à erosão e pode ser utilizada de forma mais intensiva, como em culturas anuais. Notou-se que a região Sul da área em estudo (Figura 3b) era a que possuía maior resistência natural à erosão, isso em virtude da combinação do tipo de solo, que na área é o LVd, caracterizado por apresentar valor elevado de tolerância à perda de solo e pelos baixos valores do fator topográfico. A região Norte (Figura 3b) foi a que exibiu a maior fragilidade de solo, devendo apresentar valores de CPp próximos a zero. Nessa região, verificou-se a predominância do grupo de Neossolos, caracterizado por valores baixos de tolerância à perda de solo, além de elevados valores do fator topográfico e da erosividade.

Pôde-se verificar que aproximadamente $6 \%$ da área em estudo apresentava perda de solo superior ao limite tolerável, o que a define como "área prioritária a ser recuperada" dentro do planejamento local. As áreas prioritárias estavam concentradas na região Norte da bacia hidrográfica, muitas das quais próximas a nascentes de água e represas, o que pode comprometer a qualidade e quantidade da água disponível nos trechos seguintes da rede hidrográfica (Figura 3c).

\section{Zoneamento ambiental}

A distribuição espacial das zonas de uso do solo na bacia hidrográfica do ribeirão Salobra está representada na figura 4.

A Zona 1 corresponde à manutenção da vegetação, áreas úmidas e nascentes, que representam 23,8 \% área. As áreas que compõem essa zona são fundamentais no equilíbrio ecológico da bacia hidrográfica. A manutenção da vegetação nativa em conjunto com a recuperação de Áreas de Preservação Permanente (APP) corresponde a um aspecto importante na atenuação do processo erosivo (Asis \& Omasa, 2007) e no funcionando de barreiras físicas naturais de lixiviação e agroquímicos (Casalí et al., 2008). Alguns estudos revelam que a degradação da vegetação ciliar pode afetar a morfologia do canal, as taxas de erosão e a deposição de sedimentos (Silva et al., 2007).

As áreas de recuperação (Zonas 2 e 3) abrangiam $7,4 \%$ da área em estudo. Assim, é fundamental a recuperação dessas áreas, haja vista os problemas que podem se intensificar em virtude da não recuperação. Entre esses problemas podem ser citados a perda de 


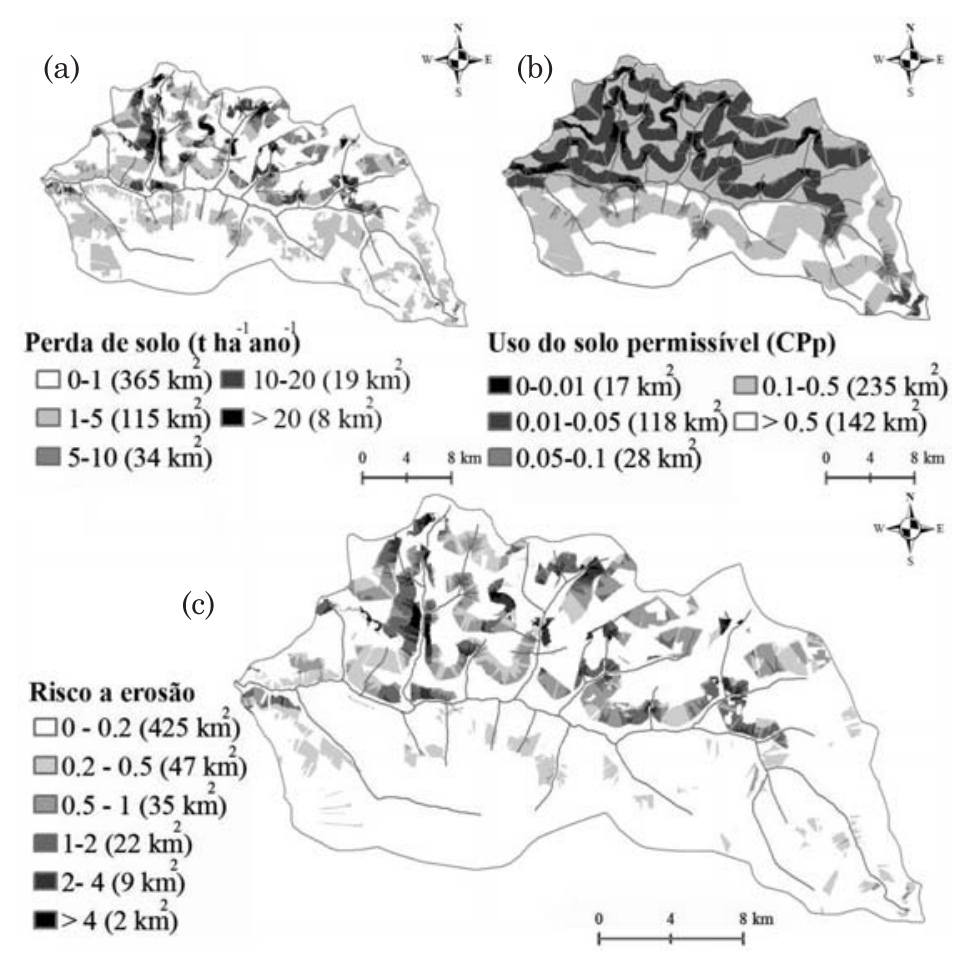

Figura 3. Perda de solo estimada pela USLE (a); Uso do solo permissível (b); e Risco de erosão (c).

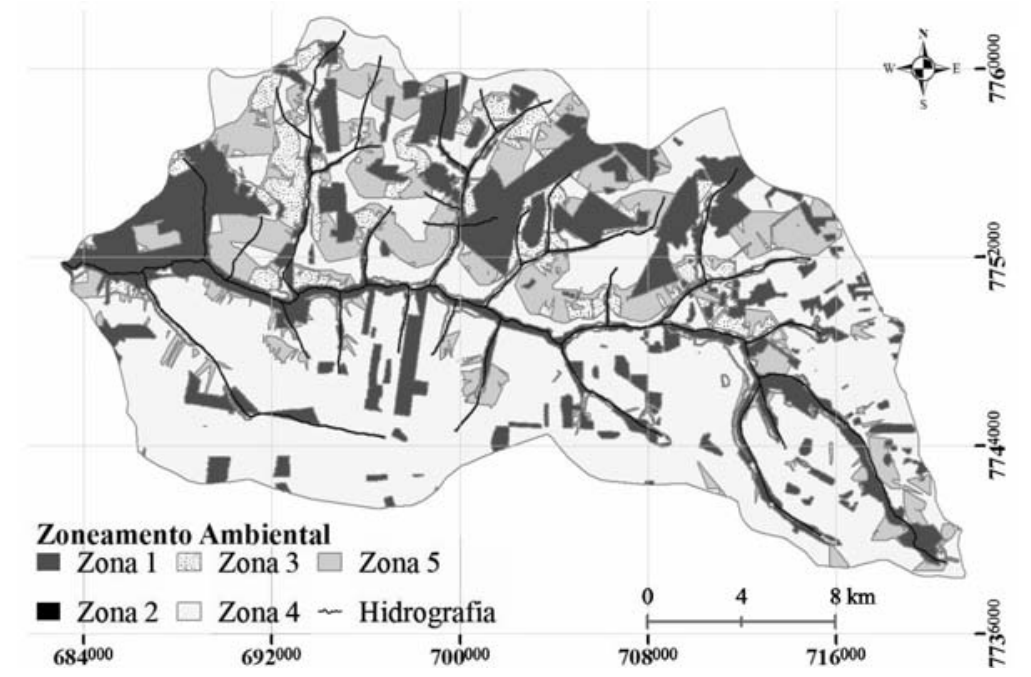

Figura 4. Zoneamento ambiental da bacia hidrográfica do ribeirão Salobra.

fertilidade do solo, o assoreamento dos córregos e o comprometimento da qualidade da água (Oliveira et al., 2011).

Na proposta de zoneamento ambiental, a área em estudo foi dividida em duas zonas de uso agrícola. A primeira, Zona 4, representava as áreas mais resistentes à erosão e correspondia a 54,8 \% da área. Nessa zona, podia-se realizar o cultivo de culturas anuais com adoção de técnicas de manejo do solo. A Zona 5 (14 \% da área), ao contrário, representava as áreas mais suscetíveis à erosão, exigindo cobertura e manejo regular do solo. Nessas áreas, recomenda-se o cultivo de culturas perenes ou pastagens com manejo regular (Oliveira et al., 2011).

Nota-se que o zoneamento ambiental aplicado possibilita o adequado ordenamento de uso do solo e, consequentemente, a identificação de irregularidades e de prioridades de conservação. Assim, esse método pode ser utilizado no contexto do planejamento estratégico, tanto da aplicação de recursos financeiros quanto de ações de intervenção, visando à gestão de bacias hidrográficas. Além disso, o zoneamento ambiental pode ser estudado, em próximas etapas, como ferramenta para norteamento de programas de 
pagamento de serviços ambientais, a exemplo do programa "Produtor de Água" (Chaves et al., 2004; ANA, 2008), que estabelece incentivos para a realização de práticas agrícolas e de preservação ambiental.

\section{Monitoramento das características quali- quantitativas da água}

$\mathrm{Na}$ quadro 4 são apresentados os modelos de regressão recomendados para representação das vazões correspondentes aos níveis de permanência de 50, 90 e 95 \%. Verificou-se que o melhor modelo, para todos os níveis de permanência, foi o potencial, utilizando área de drenagem. Além disso, a região foi definida como hidrologicamente homogênea, pois obtiveram-se valores adequados para todos os parâmetros avaliados.

As equações de regionalização obtidas foram utilizadas para o cálculo dos valores de permanência para a seção na foz do ribeirão Salobra. Assim, utilizando-se do valor de área da bacia hidrográfica, foi possível estimar as vazões de permanência e, posteriormente, o cálculo dos coeficientes a e b da equação 10 (Quadro 5).
Quadro 5. Valores utilizados na obtenção da curva de permanência para a seção em estudo

\begin{tabular}{cccccc}
\hline Área & $\mathbf{Q}_{\mathbf{5 0}}$ & $\mathbf{Q}_{\mathbf{9 0}}$ & $\mathbf{Q}_{\mathbf{9 5}}$ & $\mathbf{a}$ & $\mathbf{b}$ \\
\hline $\mathrm{km}^{2}$ & & & & & \\
540 & 6,55 & 5,52 & 5,02 & $-0,590001$ & 1,584482 \\
\hline
\end{tabular}

A equação utilizada para regionalizar a curva de permanência na seção de estudo pode, desse modo, ser escrita conforme a equação 13 .

$$
\mathrm{Q}=\exp (-0,590001 \mathrm{P}+1,584482)
$$

em que $\mathrm{P}=$ permanência (0 a 1$)$.

A partir da equação 13 foram obtidas a curva de permanência e, posteriormente, as curvas TMDL de fósforo total (ambiente lótico) e nitrato da foz do ribeirão Salobra (Figura 5).

A partir das informações da figura 5, podem-se obter valores de TMDL para as diversas vazões de permanência, possibilitando avaliar a qualidade da água do rio nas vazões ao longo do ano. Além disso, é possível verificar as contribuições específicas por área,

Quadro 4. Modelos de regressão recomendados para a estimativa das vazões $\mathbf{Q}_{50}, \mathbf{Q}_{90}$ e $\mathbf{Q}_{95}$

\begin{tabular}{lccc}
\hline Permanência & $\mathbf{Q}_{\mathbf{5 0}}$ & $\mathbf{Q}_{\mathbf{9 0}}$ & $\mathbf{Q}_{\mathbf{9 5}}$ \\
\hline \% & & & \\
Modelo potencial & $\mathrm{Q}_{50}=0,033517 \mathrm{~A}^{0,838453}$ & $\mathrm{Q}_{90}=0,030323 \mathrm{~A}^{0,776202}$ & $\mathrm{Q}_{95}=0,024946 \mathrm{~A}^{0,780915}$ \\
$\mathrm{R}^{2}$ & 0,97436 & 0,92462 & 0,90631 \\
$\mathrm{R}^{2}$ ajustado & 0,96153 & 0,88693 & 0,85946 \\
Teste F (valor de p) & 0,01291 & 0,02843 & 0,03800 \\
\hline
\end{tabular}

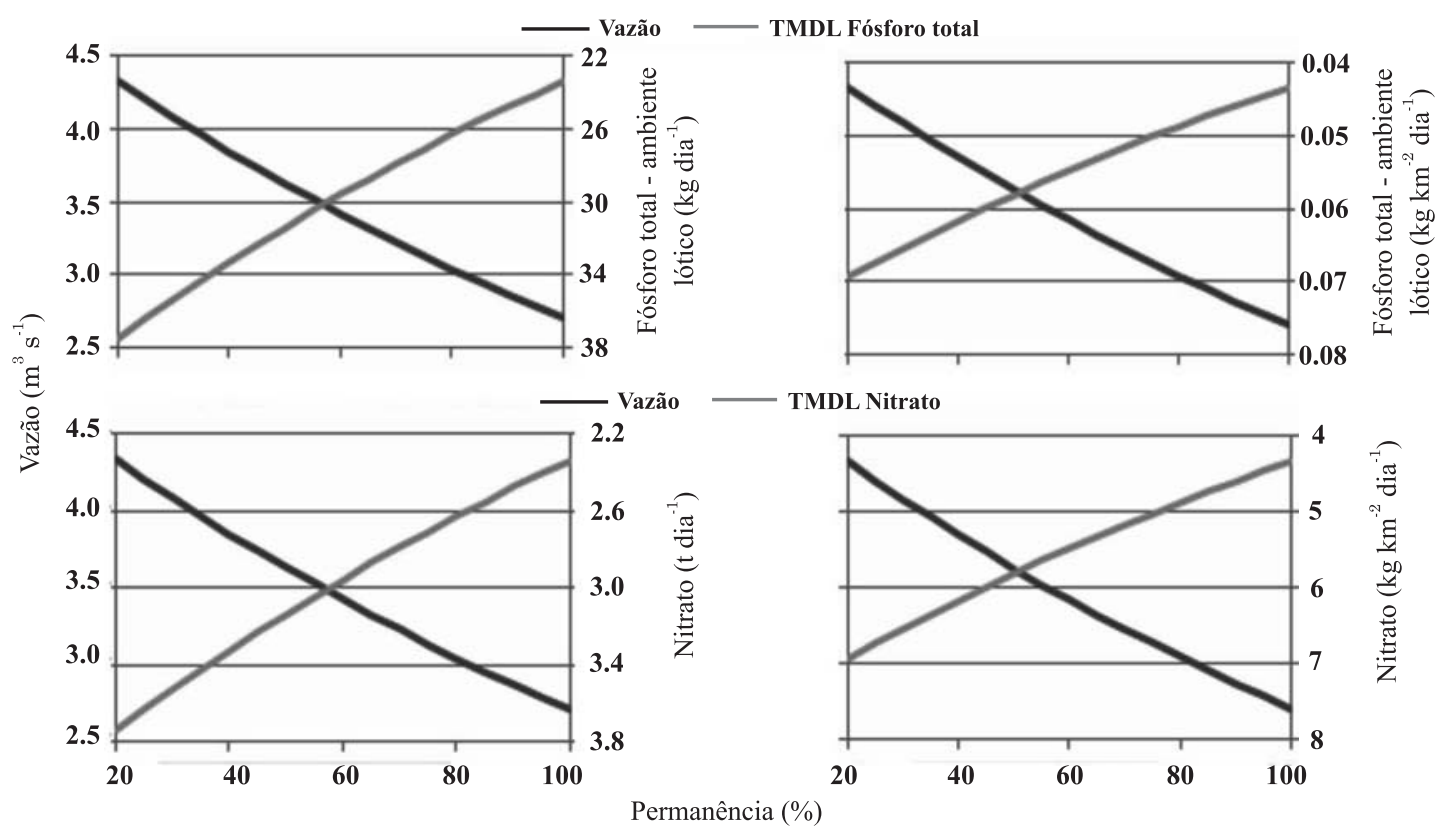

Figura 5. Curvas TMDL. 
ou seja, podem-se avaliar cargas de poluição pontual e difusa. Assim, tomando como exemplo uma vazão $\mathrm{Q}_{60}$ igual a $3,4 \mathrm{~m}^{3} \mathrm{~s}^{-1}$, podem-se obter os valores da carga máxima permitida de fósforo total corresponde a $29,6 \mathrm{~kg} \mathrm{dia}^{-1}\left(0,055 \mathrm{~kg} \mathrm{~km}^{-2} \mathrm{dia}^{-1}\right)$ e de nitrato de $2,96 \mathrm{t} \mathrm{dia}^{-1}\left(5,48 \mathrm{~kg} \mathrm{~km}^{-2} \mathrm{dia}^{-1}\right)$ (Figura 5).

A partir dos valores da curva de permanência e de padrões de qualidade da água, é possível desenvolver novas curvas TMDL. No Brasil, ainda não há padrões para material sólido em suspensão transportado em rios, o que dificulta o monitoramento de sedimentos erodidos nas bacias hidrográficas. É fundamental a realização de estudos de transporte de sedimentos, para serem propostos valores-padrão de transporte de sedimentos por bacia hidrográfica. Desse modo, tornase possível monitorar, de forma completa, as alterações de qualidade e quantidade de água que o uso e manejo do solo promovem nos recursos hídricos.

\section{CONCLUSÕES}

1. O zoneamento ambiental aplicado permite a determinação de zonas de uso do solo e, consequentemente, a identificação de irregularidades e de prioridades de conservação. Pode ser usado para a melhor distribuição dos recursos financeiros nas unidades de gestão ambiental e melhoria das condições de conservação e preservação e da produção agrícola.

2. As áreas que apresentam perda de solo superior ao limite tolerável correspondem a aproximadamente 6\% da área em estudo, o que a define como a área prioritária a ser recuperada dentro do planejamento ambiental.

3. As curvas TMDL podem ser utilizadas para auxiliar no monitoramento e fiscalização da qualidade da água do ribeirão Salobra, pois possibilitam a análise da carga total máxima diária permitida de poluentes em condições críticas do corpo d'água. Além disso, o método é simples e os resultados são facilmente aplicados, o que possibilita o seu uso prático no âmbito da gestão das águas.

\section{AGRADECIMENTOS}

Ao Conselho Nacional de Desenvolvimento Científico e Tecnológico (CNPq) e à Fundação de Apoio ao Desenvolvimento do Ensino, Ciência e Tecnologia do Estado de Mato Grosso do Sul (FUNDECT), pelo auxílio financeiro para a realização deste trabalho.

\section{LITERATURA CITADA}

AGÊNCIA NACIONAL DE ÁGUAS - ANA. HidroWeb, Sistema de informações hidrológicas. Disponível em: <http:// hidroweb.ana.gov.br/>. Acesso em: 24 nov. de 2010.
AGÊNCIA NACIONAL DE ÁGUAS - ANA. Manual operativo do Programa Produtor de Água. Brasília, Superintendência de Usos Múltiplos, 2008.

AMORE, E.; MODICA, C.; NEARING, M.A. \& SANTORO, V.C. Scale effect in USLE and WEPP application for soil erosion computation from three Sicilian basins. J. Hydrol., 293:100-114, 2004.

ASIS, A.M. \& OMASA, K. Estimation of vegetation parameter for modeling soil erosion using linear Spectral Mixture Analysis of Landsat ETM data. ISPRS J. Photogram. Remote Sens., 62:309-324, 2007.

BASIC, F.; KISIC, I.; MESIC, M.; NESTROY, O. \& BUTORAC, A. Tillage and crop management effects on soil erosion in central Croatia. Soil Tillage Res, 78:197-206, 2004.

BESKOW, S.; MELLO, C.R.; NORTON, L.D.; CURI, N.; VIOLA, M.R. \& AVANZI, J.C. Soil erosion prediction in the Grande River Basin, Brazil using distributed modeling. Catena, 79:49-59, 2009.

BHATTACHARYYA, P.; BHATT, V.K. \& MANDAL, D. Soil loss tolerance limits for planning of soil conservation measures in Shivalik-Himalayan region of India. Catena, 73:117-124, 2008 .

BORSUK, M.; STOW, C. \& RECKHOW, K.H. Predicting the frequency of water quality standard violations: A probabilistic approach for TMDL development. Environ. Sci. Technol., 36:2109-2115, 2002.

BRASIL. Conselho Nacional de Meio Ambiente. Resolução CONAMA n. 357. Dispõe sobre a classificação dos corpos de água e diretrizes para o seu enquadramento, bem com estabelece as condições e padrões de lançamento de efluentes, e dá outras providências. Brasília, 2005. Diário Oficial [da] União, 18 mar. 2005.

BRASIL. Conselho Nacional de Meio Ambiente. Resolução CONAMA n ${ }^{\circ} 302$. Dispõe sobre os parâmetros, definições e limites de Áreas de Preservação Permanente de reservatórios artificiais e o regime de uso do entorno. Brasília, 2002a. Diário Oficial [da] União, 20 de mar. 2002a.

BRASIL. Conselho Nacional de Meio Ambiente. Resolução CONAMA n ${ }^{\circ} 303$. Dispõe sobre parâmetros, definições e limites de Áreas de Preservação Permanente. Brasília, 2002b. Diário Oficial [da] União, 20 de mar. 2002b.

BRASIL. Lei no 4.771. Institui o novo Código Florestal. Brasília, 1965. Diário Oficial [da] União, 15 de dezembro 1965.

BRASIL. Ministério das Minas e Energia. Projeto RADAMBRASIL. Folha SF.21 Campo Grande: Geologia, geomorfologia, pedologia, vegetação e uso potencial da terra. Rio de Janeiro, 1982. 412p. (Levantamento de Recursos Naturais, 28)

CÂMARA, G.; SOUZA, R.C.M.; FREITAS, U.M. \& GARRIDO, J. SPRING: Integrating remote sensing and GIS by object-oriented data modelling. Comp. Graphics, 20:395403, 1996.

CASALÍ, J.; GASTESI, R.; A’LVAREZ-MOZOS, J.; DE SANTISTEBAN, L.M.; DEL VALLE DE LERSUNDI, J.; GIME'NEZ, R.; LARRAÑAGA, A.; GOÑI, M.; AGIRRE, U.; CAMPO, M.A.; LÓPEZ, J.J. \& DONÉZAR, M. Runoff, erosion, and water quality of agricultural watersheds in central Navarre (Spain). Agric. Water Manag., 95:11111128, 2008. 
CHAVES, H.M.L.; BRAGA JR., B.; DOMINGUES, A.F. \& SANTOS, D.G. Quantificação dos benefícios ambientais e compensações financeiras do "Programa do Produtor de Água" (ANA): I.Teoria. R. Bras. Rec. Hídricos, 9:5-14, 2004.

DESMET, P.J.J \& GOVERS, G. A GIS-procedure for automatically calculating the USLE LS-factor on topographically complex landscape units. J. Soil Water Conserv., 51:427-433, 1996.

DIRETORIA DO SERVIÇO GEOGRÁFICO BRASILEIRO DSG. Carta Campo Grande. Folha SF. 21-X-B-II. Escala 1:100.000. Ministério do Exército. Região Centro-Oeste do Brasil. Carta, 1979.

DIRETORIA DO SERVIÇO GEOGRÁFICO BRASILEIRO DSG. Carta Palmeiras. Folha SF. 21. X. B. III. Escala 1:100.000. Ministério do Exército. Região Centro-Oeste do Brasil. Carta, 1988.

ENVIRONMENTAL SYSTEMS RESEARCH INSTITUTE ESRI. ArcGIS Professional GIS for the desktop, version 9.2. Software. 2006.

FERRO, V. Deducing the USLE mathematical structure by dimensional analysis and self-similarity theory. Biosyst. Eng., 106:216-220, 2010.

GABRIELS, D.; GHEKIERE, G.; SCHIETTECATTE, W. \& ROTTIERS, I. Assessment of USLE cover-management C-factors for 40 crop rotation systems on arable farms in the Kemmelbeek watershed, Belgium. Soil Tillage Res, 74:47-53, 2003.

HAVENS, K.E. \& SCHELSKE, C.L. The importance of considering biological processes when setting total maximum daily loads (TMDL) for phosphorus in shallow lakes and reservoirs. Environ. Pollut., 113:1-9, 2001.

INSTITUTO NACIONAL DE PESQUISAS ESPACIAIS INPE. Imagem LANDSAT 5 Thematic Mapper (TM), canais $1,2,3,4,5,6,7$, órbita 225 , ponto 74 , de 18 dezembro 2008. São José dos Campos, Instituto Nacional de Pesquisas Espaciais, Disponível em: http:// www.dgi.inpe.br/CDSR/. Acesso em: 17 mar. 2009.

KANG, M.S.; PARK, S.W.; LEE, J.J. \& YOO, K.H. Applying SWAT for TMDL programs to a small watershed containing rice paddy fields. Agric. Water Manag., 79:7292, 2006.

KINNELL, P.I.A. Event soil loss, runoff and the Universal Soil Loss Equation family of models: A review. J. Hydrol., 385:384-397, 2010

MOEHANSYAH, H.; MAHESHWARI, B.L. \& ARMSTRONG, J. Field evaluation of selected soil erosion models for catchment management in Indonesia. Biosyst. Eng., 88:491-506, 2004.

MORGAN, R.P.C. A simple approach to soil loss prediction: A revised Morgan-Morgan-Finney model. Catena, 44:305322, 2001.

MORGAN, R.P.C.; QUINTON, J.N.; SMITH, R.E.; GOVERS, G.; POESEN, J.W.A.; AUERSWALD, K.; CHISCI, G.; TORRI, D. \& STYCZEN, M.E. The European Soil Erosion Model (EUROSEM): A dynamic approach for predicting sediment transport from ûelds and small catchments. Earth Surf. Proc. Landforms, 23:527-544, 1998.
NEARING, M.A.; FOSTER, G.R.; LANE, L.J. \& FINKNER, S.C. A process based soil erosion model for USDA Water Erosion Prediction Project technology. Trans. ASAE, 32:1587-1593, 1989.

OGG, C.W. \& KEITH, G.A. New federal support for priority watershed management needs. J. Am. Water Res. Assoc., 38:577-586, 2002

OLIVEIRA, P.T.S. Zoneamento ambiental no planejamento e gestão de bacias hidrográficas. Campo Grande, Universidade Federal do Mato Grosso do Sul, 2011. 78p. (Tese de Mestrado)

OLIVEIRA, P.T.S.; ALVES SOBRINHO, T.; RODRIGUES, D.B.B. \& PANACHUKI, E. Erosion risk mapping applied to environmental zoning. Water Res. Manag., 25:10211036, 2011.

OLIVEIRA, P.T.S.; RODRIGUES, D.B.B.; ALVES SOBRINHO, T. \& PANACHUKI, E. Estimativa do fator topográfico da USLE a partir de três algoritmos. Ambi-Agua, 5:217-225, 2010 .

PLANO DE CONSERVAÇÃO DA BACIA DO ALTO PARAGUAI - PCBAP. Projeto Pantanal. Programa Nacional do Meio Ambiente. Metodologia do Plano de Conservação da Bacia do Alto Paraguai. Brasília, MMA/ SEMAM/PNMA, 1997

RENARD, K.G.; FOSTER, G.R.; WEESIES, G.A.; MCCOOL, D.K. \& YODER, D.C. Predicting soil erosion by water: A guide to conservation planning with the revised Universal Soil Loss Equation (RUSLE). Washington, USDA, 1997. (Agriculture Handbook, 703)

RISSE, L.M.; NEARING, M.A.; NICKS, A.D. \& LAûEN, J.M Error assessment in the universal soil loss equation. Soil Sci. Soc. Am. J., 57:825-833, 1993.

SILVA, A.M.; CASATTI, L.; ÁLVARES, C.A.; LEITE, A.M.; MARTINELLI, L.A. \& DURRANT, S.F. Soil loss risk and habitat quality in streams of a meso-scale river basin. Sci Agric, 64:336-343, 2007.

SOUSA, H.T. Sistema computacional para regionalização de vazões. Viçosa, MG, Universidade Federal de Viçosa, 2009. 86p. (Tese de Mestrado)

STRINGFELLOW, W.; HERR, J.; LITTON, G.; BRUNELL, M.; BORGLIN, S.; HANLON, J.; CHEN, C.; GRAHAM, J.; BURKS, R.; DAHLGREN, R.; KENDALL, C.; BROWN, R. \& QUINN, N. Investigation of river eutrophication as part of a low dissolved oxygen total maximum daily load implementation. Water Sci. Technol., 59:9-14, 2009.

TIWARI, A.K.; RISSE, L.M. \& NEARING, M. Evaluation of WEPP and its comparison with USLE and RUSLE. Trans. ASAE, 43:1129-1135, 2000.

TUCCI, C.E.M. Hidrologia, ciência e aplicação. 3.ed. Porto Alegre, Associação Brasileira de Recursos Hídricos, 2003. 943p.

TUCCI, C.E.M. Regionalização de Vazões. Porto Alegre, Universidade Federal do Rio Grande do Sul, 2002. 256p.

WEILL, M.A.M. \& SPAROVEK, G. Estudo da erosão na microbacia do Ceveiro (Piracicaba, SP): I - Estimativa das taxas de perda de solo e estudo de sensibilidade dos fatores do modelo EUPS. R. Bras. Ci Solo, 32:801-814, 2008. 
WISCHMEIER, W.H. \& SMITH, D.D. Predicting rainfall erosion losses. A guide to conservation planning. Washington, USDA, 1978. 58p. (Agriculture Handbook, 537)

WOOLHISER, D.A.; SMITH, R.E. \& GOODRICH, D.C. KINEROS: A kinematic runoff and erosion model: Documentation and user manual. Washington, U.S. Department of Agriculture, 1990. 130p. (Agricultural Research Service, ARS-77)
ZHANG, H. \& YU, S. Critical flow-storm approach to total maximum daily load (TMDL) development: An analytical conceptual model. Front. Environ. Sci. Eng. Chin., 2:267273, 2008.

ZHANG, X.; SHAO, M.; LI, S. \& PENG, K. A review of soil and water conservation in China. J. Geogr. Sci, 14:259-274, 2004 . 